\title{
Deutscher Bibliotheksverband begrüßt anlässlich der Einigung auf eine Regierungsbildung die bibliothekspolitischen Aussagen im Koalitionsvertrag und fordert deren zügige Umsetzung
}

http://doi.org/10.1515/bd-2018-0049

Der Deutsche Bibliotheksverband e. V. (dbv) begrüßt anlässlich der bevorstehenden Regierungsbildung die im Entwurf des Koalitionsvertrags getroffenen bibliothekspolitischen Aussagen ausdrücklich. Diese eröffnen den Bibliotheken weitere Perspektiven auch auf bundespolitischer Ebene. Besonders erfreulich ist, dass geprüft werden soll, „wie der Bund zum Erhalt der vielfältigen Bibliothekslandschaft und ihrer zunehmend gesellschaftlichen Bedeutung beitragen kann. Bibliotheken sollten auch im digitalen Zeitalter ihre zentralen Funktionen für Bildung und Kultur erfüllen können,“ so der Text im Koalitionsvertrag weiter.

Die rechtliche Gleichstellung von E-Books und gedruckten Büchern ist eine der Kernforderungen des Verbandes, da Bibliotheken auch im digitalen Umfeld ihrem Auftrag nachkommen müssen, Teilhabe und Zugang zu Informationen zu gewähren. Insofern begrüßt der dbv, dass der Bund sich dafür einsetzen möchte, den „Bibliotheksnutzern unter Wahrung der Vertragsfreiheit ein noch besserer Zugang zum Repertoire von E-Books zu ermöglichen“.

Die Koalitionsparteien wollen ein gesamtstaatliches Bündnis für kulturelle Bildung und Vermittlung sowie Medienkompetenz schließen, um den Zugang zu Kunst, Kultur, Bildung und Medien zu stärken. Auch wird mit Bundesmitteln die Initiative „Kultur macht stark. Bündnisse für Bildung“ mit außerschulischen Angeboten in $\mathrm{u}$.a. Bibliotheken weiterhin unterstützt, an der auch der dbv seit 2013 aktiv beteiligt ist. Mit dem seit Beginn 2018 gestarteten neuen Projekt „Total Digital“ leisten die Bibliotheken einen weiteren Beitrag, der kulturelle Bildung für bildungsferne Kinder und Jugendliche ermöglichen soll.

„Ich freue mich, dass der Bund der herausragenden gesellschaftlichen Bedeutung von Bibliotheken Rechnung trägt und prüfen möchte, wie er zum Erhalt der vielfältigen Bibliothekslandschaft beitragen kann. Wichtig ist jedoch auch, dass der Bund durch eine aktuelle Gesetzgebung und notwendige nationale Strategien sowie bundesweite Projektförderung die zeitgemäßen Bibliothek- 
sangebote unterstützt und wichtige Impulse für eine moderne Bibliotheksentwicklung setzt“, so die Bundesvorsitzende Barbara Lison.

Bibliotheken sind als Teil von Wissenschaftseinrichtungen wichtige Akteure der digitalen Transformation des wissenschaftlichen Publikationsmarktes und im Verbund mit anderen Einrichtungen wesentliche Träger von Informationsinfrastrukturen für die Forschung. Daher begrüßt der dbv die geplante Entwicklung einer nationalen Open-Access-Strategie und einer nationalen Forschungsdaten-Infrastruktur.

Für die Bibliotheken ist auch das Bekenntnis zur Fortführung der Programme zum Erhalt des schriftlichen Kulturgutes von großer Bedeutung. Die vom dbv lange geforderte umfassende nationale Digitalisierungsstrategie des Bundes soll jetzt entwickelt werden; sie soll auch eine mit substanziellen finanziellen Mitteln unterlegte Strategie für die Zukunft von Kultureinrichtungen und ihre digitale Transformation umfassen. So wird die wichtige Funktion der Deutschen Digitalen Bibliothek zur Sicherung unseres kulturellen Erbes unterstützt, die einer zuverlässigen und nachhalten Bund-Länder-Förderung bedarf.

Die Vize-Bundesvorsitzende des dbv, Beate Tröger: „Die nur bis zum Jahr 2023 geltenden Regelungen im Urheberrechts-Wissensgesellschafts-Gesetz für den Bildungs- und Wissenschaftsbereich müssen verstetigt werden. Auch die Weiterentwicklung des Urheberrechts auf europäischer Ebene muss unter Berücksichtigung der Bedarfe von Wissenschaft und Forschung entwickelt werden. Die wissenschaftlichen Bibliotheken in Deutschland stehen im digitalen Zeitalter vor großen Herausforderungen, die sie gemeinsam mit politischen Entscheidungsträgern und Mittelgebern gestalten möchten.“

\section{Kontakt: Deutscher Bibliotheksverband e. V.}

Maiken Hagemeister, Pressesprecherin und Leitung Kommunikation, Tel.: 0 30/644989925

E-Mail: hagemeister@bibliotheksverband.de, http://www.bibliotheksverband. de, http://www.bibliotheksportal.de 